\title{
Trastuzumab Emtansine-Induced Nodular Regenerative Hyperplasia: Is Dose Reduction Enough as a Preventable Measure?
}

\author{
Isabel Garrido ${ }^{a, b}$ Adriana Magalhães ${ }^{c}$ Joanne Lopes ${ }^{d}$ Guilherme Macedo ${ }^{a, b}$ \\ aGastroenterology and Hepatology Department, Centro Hospitalar Universitário de São João, Porto, Portugal;

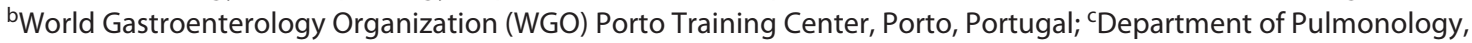

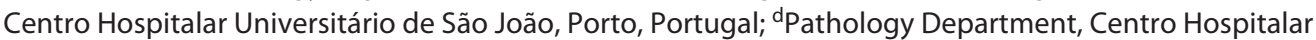 \\ Universitário de São João, Porto, Portugal
}

\section{Keywords}

Trastuzumab emtansine $\cdot$ Lung cancer $\cdot$ Nodular regenerative hyperplasia

\begin{abstract}
Introduction: Trastuzumab emtansine (T-DM1) is a novel antibody-drug conjugate targeting the human epidermal growth factor receptor 2 , used in some recurrent metastatic cancers. It was linked to modest increases in serum aminotransferase elevations and bilirubin. More recently, some cases of noncirrhotic portal hypertension have been described in patients on long-term T-DM1. The underlying liver condition is usually nodular regenerative hyperplasia (NRH) with elements of sinusoidal obstruction. Case Report: We report the case of a 52-year-old woman who started T-DM1 therapy for recurrent metastatic lung adenocarcinoma. Although a progressive reduction in lung nodules was noticed, there was a new-onset cytocholestasis and elevation in bilirubin. A reduction in platelet count was also apparent over several months during the T-DM1 therapy. Liver biopsy revealed NRH and so the dose of T-DM1 was reduced. Thereafter, the patient had normalization of liver tests and platelet count. T-DM1 was continued for more than 9 months with no signs of portal hypertension or cancer progression. Conclusions: We presented a rare case of $\mathrm{NRH}$ induced by T-DM1
\end{abstract}

Karger@karger.com

(c) 2022 S. Karger AG, Basel

www.karger.com/ddi

Karger' in a patient with lung adenocarcinoma. A high index of suspicion for liver injury and NRH must be maintained for patients who develop liver test abnormalities and/or signs of portal hypertension during treatment with T-DM1. This is the first report of a successful dose reduction in a patient with $\mathrm{NRH}$ induced by T-DM1, suggesting that it is possible to maintain the drug while it is being effective for lung cancer treatment.

(c) 2022 S. Karger AG, Basel

\section{Introduction}

Trastuzumab emtansine (T-DM1) is a novel drug composed of trastuzumab, a monoclonal antibody that targets the human epidermal growth factor receptor 2 (HER2), SMCC, a thioether linker, and emtansine (DM1), a microtubule inhibitor [1]. Once bound to HER2, T-DM1 is internalized and undergoes proteolytic digestion, releasing the cytotoxic microtubule inhibitor within the target cells. Due to emtansine, T-DM1 extends the trastuzumab-mediated effects in blocking HER2-mediated signal transduction and facilitates antibody-dependent cellmediated cytotoxicity. Therefore, T-DM1-based regimens are promising and offer the potential for better efficacy in the treatment of HER2-positive lung cancer [2]. 


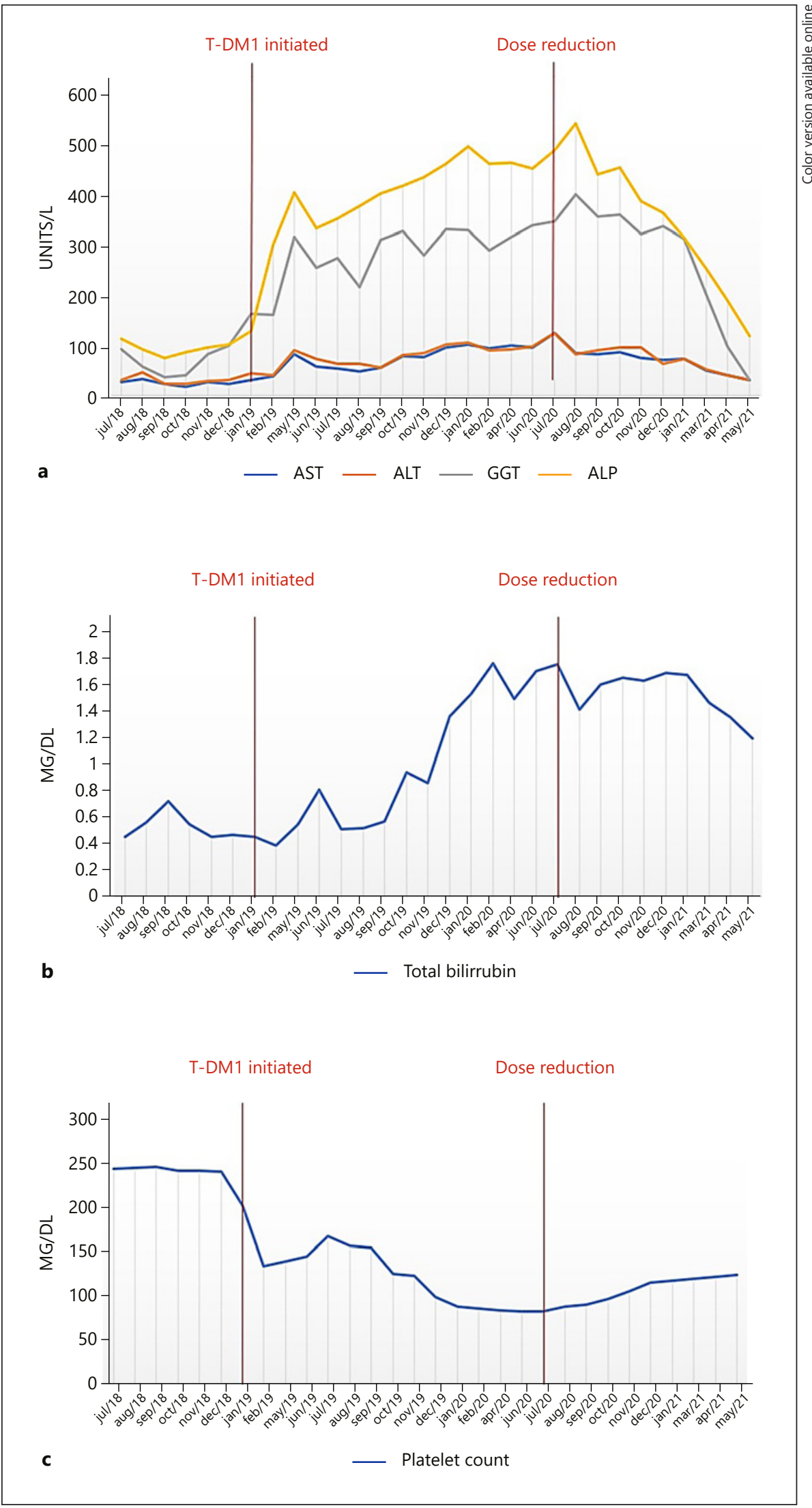

Fig. 1. Evolution of liver function tests (a), total bilirubin (b) and platelets (c) during T-DM1 treatment. AST, aspartate aminotransferase; ALT, alanine aminotransferase; GGT, gamma-glutamyl transferase; ALP, alkaline phosphatase; T-DM1, trastuzumab emtansine. 
On the other hand, several studies have shown associated adverse events that can result in drug withdrawal [3]. Thrombocytopenia is one of the most frequently cited. Moreover, T-DM1 was linked to serum transaminases elevation in up to $20-80 \%$ of patients. More recently, a few cases of noncirrhotic portal hypertension have also been described [4]. Interestingly, in those who undergo liver biopsy, cirrhosis is not present. Instead, the underlying liver condition is nodular regenerative hyperplasia (NRH). T-DM1-induced NRH usually requires a definitive interruption of the therapy. Herein, we report a case of a patient with lung adenocarcinoma who received T-DM1 therapy and developed NRH-related cholestasis, gradually improving after drug dose reduction.

\section{Case Report}

A 52-year-old woman, with a past medical history of iliofemoral deep venous thrombosis due to factor V Leiden thrombophilia under warfarin treatment, was diagnosed with lung adenocarcinoma in 2016 (stage pT3N1R0) with high PD-L1 expression and HER2-positive. She underwent right middle lobectomy and adjuvant chemotherapy (vinorelbine and carboplatin). A year later, cancer recurrence occurred, with pulmonary metastasis in the right upper lobe, and, therefore, radiotherapy was initiated. However, a progression of the disease with bilateral pulmonary metastasis was noticed and, thus, she started treatment with pembrolizumab for 6 cycles. Pulmonary lesions worsened, so the treatment was switched to afatinib in July 2018. She had cutaneous toxicity with afatinib and, thereafter, hemolytic uremic syndrome with docetaxel. In January 2019, the patient started T-DM1 $3.6 \mathrm{mg} / \mathrm{kg}$ every 3 weeks.

Multiple thoracic computed tomography (CT) scans were performed during the treatment with T-DM1, which showed a progressive reduction of lung nodules. However, we noticed new-onset cytocholestasis (Fig. 1a) and subsequent elevation in bilirubin (Fig. 1b). Before initiating T-DM1, liver function tests were normal. A reduction in platelet count was also apparent over several months during the T-DM1 therapy (Fig. 1c).

The patient denied alcohol abuse, blood transfusion, high-risk sexual behaviors, tattoos, intravenous drug use, or consumption of herbal preparations. Serologic tests for acute and chronic viral hepatitis were negative. Ceruloplasmin, alpha-1 antitrypsin, and iron tests were normal. Autoimmune serologies were also negative. CT scan revealed a liver with normal morphology and without hepatic metastasis, portal thrombosis, or splenomegaly. Magnetic resonance cholangiopancreatography showed normal intrahepatic and extrahepatic bile ducts. Upper gastrointestinal endoscopy ruled out gastroesophageal varices or other stigmata of portal hypertension. Transient elastography was also performed, revealing a value of $5.5 \mathrm{kPa}$.

The patient had no symptoms during this time. Nonetheless, liver function seemed to get worse (aspartate aminotransferase 105 $\mathrm{U} / \mathrm{L}$, alanine aminotransferase $110 \mathrm{U} / \mathrm{L}$, gamma-glutamyl transferase $338 \mathrm{U} / \mathrm{L}$, alkaline phosphatase $474 \mathrm{U} / \mathrm{L}$, total bilirubin 1.75 $\mathrm{mg} / \mathrm{dL})$, as did the platelet count $\left(74 \times 10^{9} / \mathrm{L}\right)$. Due to the patient's

Trastuzumab Emtansine-Induced

Nodular Regenerative Hyperplasia
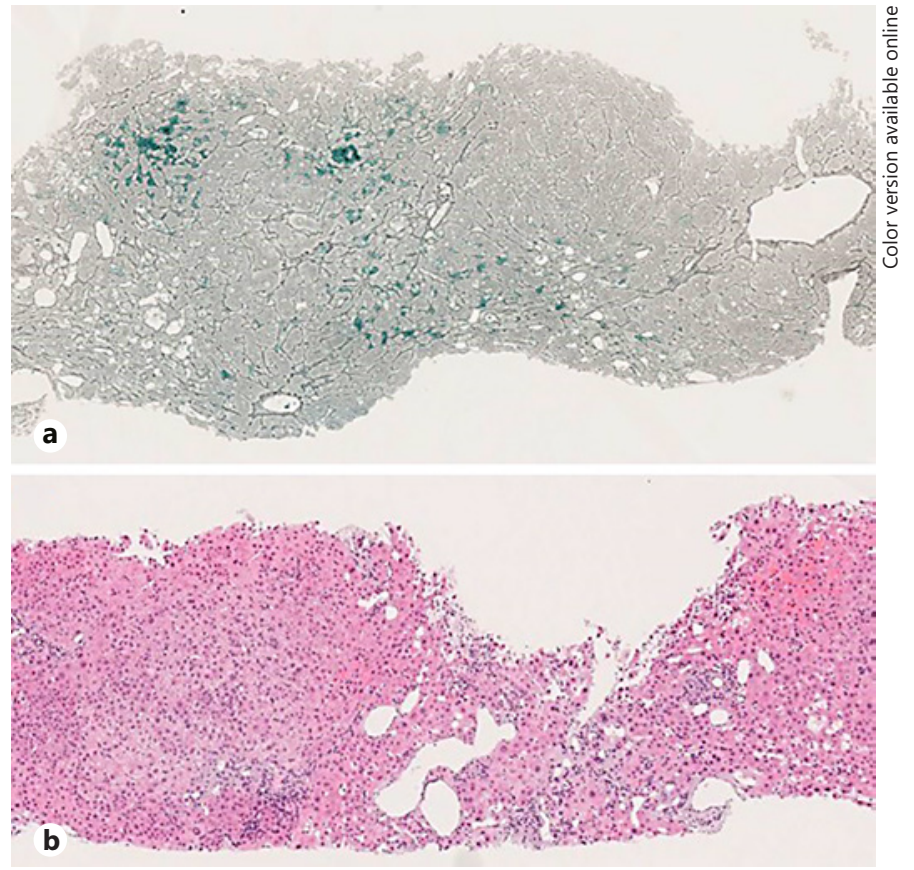

Fig. 2. Reticulin (a) and HE stain (b): Diffuse nodules of hyperplastic hepatocytes compressing adjacent single cell plates and sinusoidal dilation. Minimal fibrosis.

prothrombotic condition, being under warfarin treatment (international normalized ratio 2.8) and low platelet count, we decided to perform a transjugular liver biopsy in July 2020. The anatomopathological study revealed an NRH (Fig. 2). Hence, the dose of T-DM1 was reduced ( $1.8 \mathrm{mg} / \mathrm{kg}$ every 3 weeks). Thereafter, liver biochemical tests had been gradually improving, as well as platelet count (Fig. 1). Up to this point, the patient had no cancer progression and no signs of portal hypertension.

\section{Discussion}

NRH is characterized by a diffuse transformation of normal hepatic parenchyma into small and regenerative nodules, with little to no fibrosis $[5,6]$. This phenomenon is the consequence of heterogeneous hepatic perfusion. The portal flow is decreased due to obliterative portal venopathy, leading to ischemia and atrophy of the irrigated hepatocytes. Consequently, the central atrophy is compensated by increased blood flow in the adjacent areas, which leads to hyperplasia of hepatocytes and the formation of regenerative nodules. No significant fibrosis is found in or around the nodules. This is an important feature distinguishing NRH from cirrhosis and from focal nodular hyperplasia of the liver. 
Table 1. Summary of previously reported cases of T-DM1-induced NRH

\begin{tabular}{|c|c|c|c|c|c|}
\hline & Force et al. [11] & Force et al. [11] & Prochaska et al. [12] & Lepelley et al. [13] & Hassan et al. [14] \\
\hline Disease & Breast cancer & Breast cancer & Breast cancer & Breast cancer & Breast cancer \\
\hline Pattern & Hepatocellular & Hepatocellular & Hepatocellular & Hepatocellular & Hepatocellular \\
\hline Thrombocytopenia & Yes & Yes & Yes & - & Yes \\
\hline Latency & 15 months (23 cycles) & 17 months (22 cycles) & 15 months (21 cycles) & 12 months (16 cycles) & 27 months (39 cycles) \\
\hline Portal hypertension & $\begin{array}{l}\text { Yes - ascites, esophageal varices, } \\
\text { hepatic encephalopathy, portal } \\
\text { vein thrombosis }\end{array}$ & Yes - esophageal varices & $\begin{array}{l}\text { Yes - splenomegaly, } \\
\text { esophageal varices }\end{array}$ & Yes - splenomegaly & $\begin{array}{l}\text { Yes - mesenteric } \\
\text { varices }\end{array}$ \\
\hline
\end{tabular}

T-DM1, trastuzumab emtansine; $\mathrm{NRH}$, nodular regenerative hyperplasia.

The existing literature on this pathological entity is predominantly composed of case reports, so the prevalence and clinical significance of NRH are uncertain [79]. Imaging modalities such as contrast-enhanced CT and magnetic resonance imaging can help identify nodular liver lesions. However, histological evaluation is the only way to make a definitive diagnosis of $\mathrm{NRH}$ and rule out conditions such as cirrhosis or hepatocellular carcinoma [5].

We report the case of a patient who developed biopsyproven NRH during the treatment with T-DM1 for lung adenocarcinoma. The timing of the liver injury, the exclusion of other causes of hepatic disease, and normalization of laboratory abnormalities after adjusting the dose make T-DM1 the most likely explanation for NRH in our patient. This is remarkable because while liver toxicity has been well described with T-DM1, NRH is rare and has been observed in 7 patients during clinical trials [10]. Moreover, since approval and wide-scale usage, there are only 5 cases of T-DM1-induced NRH reported in the literature (Table 1) [11-14]. It should be noted that all the cases described occurred in patients with breast cancer. This is the first case report of a patient who developed $\mathrm{NRH}$ during the treatment for lung cancer. This can be justified by the fact that this drug was only recently started to be used in the treatment of lung cancer, while it has been used for the treatment of advanced HER-2 positive metastatic breast cancer since 2013 [15].

The risk of TDM-1 associated liver injury may be increased in patients with extensive prior treatment, as in our case. However, Prochaska et al. [12] showed that the risk is present even in individuals who have not had any prior therapy concerning the treatment of metastatic disease. Serum transaminases elevation was present in every case of T-DM1-induced NRH. However, our patient had a cholestatic pattern, which was rarely elevated in the abovementioned reports. Given no obvious bile duct obstruction and the temporal association with T-DM1 initiation, we assume that it was responsible for cholestasis. Indeed, the most common feature associated with $\mathrm{NRH}$ is cholestatic liver function [16]. With regard to thrombocytopenia, it was present in most cases, as in ours. Notwithstanding, since T-DM1 is known to cause thrombocytopenia in $31 \%$ of the patients, this adverse effect might not be directly related to NRH. Instead, there may be hematological toxicity of T-DM1 through impairment of megakaryocyte differentiation [14].

$\mathrm{NRH}$ is usually not diagnosed rapidly, as there is often a latent phase of asymptomatic liver damage with a mild increase of the transaminases [6]. It is usually diagnosed later, when significant portal hypertension and its complications, especially splenomegaly, ascites, and variceal bleeding, appear. The origin of portal hypertension is primarily sinusoidal, similar to that seen with cirrhosis, which may be due to the compression of the intrahepatic portal radicles by the regenerating nodules [17]. All the published cases of NRH caused by T-DM1 have reported clinical or radiographic signs of portal hypertension. In our case, we diagnosed NRH prior to the development of portal hypertension, which allowed us to adjust the therapy in a timely manner. Indeed, a high index of suspicion for drug-induced NRH must be maintained. We suggest that patients undergoing treatment with T-DM1 should be closely monitored, including regular evaluation of liver function tests, platelet count, and periodic imaging examinations. In case of abnormalities in liver function tests and/or evidence of portal hypertension, a liver biopsy should be performed. According to the clinical condition, drug discontinuation or dose reduction should be considered.

$\mathrm{NRH}$ is usually associated with systemic diseases such as rheumatologic, vascular, and myeloproliferative disor- 
ders, as well as certain metabolic diseases [5]. Drugs have also been associated with the development of this pathology [9]. Azathioprine, a purine analog, is the most commonly related drug. In fact, $\mathrm{NRH}$ is increasingly recognized in thiopurine-treated patients with inflammatory bowel disease or those undergoing liver transplantation $[18,19]$. Similar to TDM-1-induced NHR cases, manifestations are highly variable (from asymptomatic to symptoms of noncirrhotic portal hypertension). The pathogenesis is poorly understood in both situations, but liver biochemistry, platelet count, and portal pressure usually normalize after drug withdrawal $[11,18]$.

To investigate T-DM1-induced hepatotoxicity, Yan et al. [20] resorted to mouse models. The authors showed that the drug was internalized into human hepatocytes once bound to the HER2 receptor. After endocytosis, T-DM1 induced apoptosis and inhibited hepatocytes' growth by mitotic arrest. In addition, necrosis and inflammation were also highlighted in mouse liver tissue after T-DM1 treatment, whereas trastuzumab induced only inflammation and no necrosis. Indeed, there are no reports of single-agent trastuzumab-related NRH. Therefore, some authors suggest that trastuzumab could be reintroduced in a patient with NRH induced by T-DM1 [14]. It is also important to note that in these mouse subjects, T-DM1 induces a dose-dependent inflammation and liver tissue necrosis [20]. In fact, T-DM1 increased serum aminotransferases, lactate dehydrogenase, and tumor necrosis factor-alpha in a dose-dependent manner. Therefore, dose reduction may be sufficient to avoid $\mathrm{NRH}$. In our case, with halving the dose of T-DM1, the liver panel has gradually returned to the normal range. Thus, it was possible to maintain the treatment that was being effective for lung cancer and which was refractory to the several therapies previously used. To our knowledge, this is the first report of a successful dose reduction in a patient with NRH induced by T-DM1.

We presented the first case of NRH induced by T-DM1 in a patient who had lung adenocarcinoma. Liver tests and platelet count have been improving several months after reducing the dose of T-DM1, while treatment was being effective to treat the tumor. Therefore, even if T-DM1 is the cause of NRH, we hypothesize that the use of a lower dose may be both safe and efficient, as demonstrated by our particular case in which prolonged cancer remission was achieved. Further studies are needed to explore the appropriate dose to prevent the development of NRH.

\section{Statement of Ethics}

Written informed consent was obtained from the patient for publication of this case report and any accompanying images. This study protocol was reviewed and approved by the Centro Hospitalar Universitário de São João Ethics Committee (ethics reference number OS 103/2021).

\section{Conflict of Interest Statement}

The authors have no conflicts of interest to declare.

\section{Funding Sources}

There is no funding to declare.

\section{Author Contributions}

Isabel Garrido drafted the manuscript. Isabel Garrido, Adriana Magalhães, Joanne Lopes, and Guilherme Macedo have critically revised and finalized the manuscript. All authors have approved the final version of the manuscript.

\section{Data Availability Statement}

All data generated or analyzed during this study are included in this article. Further inquiries can be directed to the corresponding author.

\section{References}

Trastuzumab Emtansine-Induced

Nodular Regenerative Hyperplasia
1 Peddi PF, Hurvitz SA. Ado-trastuzumab emtansine (T-DM1) in human epidermal growth factor receptor 2 (HER2)-positive metastatic breast cancer: latest evidence and clinical potential. Ther Adv Med Oncol. 2014;6(5):2029.

2 Li BT, Shen R, Buonocore D, Olah ZT, Ni A, Ginsberg MS, et al. Ado-trastuzumab emtansine for patients with HER2-mutant lung cancers: results from a phase II basket trial. J Clin Oncol. 2018;36(24):2532-7.
3 Endo Y, Mohan N, Dokmanovic M, Wu WJ. Mechanisms contributing to ado-trastuzumab emtansine-induced toxicities: a gateway to better understanding of ADC-associated toxicities. Antib Ther. 2021;4(1):55-9.

4 LiverTox: clinical and research information on drug-induced liver injury. Bethesda, MD: National Institute of Diabetes and Digestive and Kidney Diseases; 2012. Available from: https: //www.ncbi.nlm.nih.gov/books/ NBK548559/. 
5 Reshamwala PA, Kleiner DE, Heller T. Nodular regenerative hyperplasia: not all nodules are created equal. Hepatology. 2006;44(1):714.

6 Hartleb M, Gutkowski K, Milkiewicz P. Nodular regenerative hyperplasia: evolving concepts on underdiagnosed cause of portal hypertension. World J Gastroenterol. 2011; 17(11):1400-9.

7 Figueiredo M, Nkuize M, Mulkay JP, De Wit S, Gomez M, Sersté T. Nodular regenerative hyperplasia in HIV-positive patients: a case series and review of the literature. Acta Gastroenterol Belg. 2017;80(1):15-9.

8 Jain P, Patel S, Simpson HN, Silver RM, Lewin $\mathrm{DN}$, Campbell RC, et al. Nodular regenerative hyperplasia of the liver in rheumatic disease: cases and review of the literature. J Investig Med High Impact Case Rep. 2021;9: 23247096211044617.

9 Ghabril M, Vuppalanchi R. Drug-induced nodular regenerative hyperplasia. Semin Liver Dis. 2014;34(2):240-5.

10 Diéras V, Harbeck N, Budd GT, Greenson JK, Guardino AE, Samant M, et al. Trastuzumab emtansine in human epidermal growth factor receptor 2-positive metastatic breast cancer: an integrated safety analysis. J Clin Oncol. 2014;32(25):2750-7.
11 Force J, Saxena R, Schneider BP, Storniolo AM, Sledge GW Jr, Chalasani N, et al. Nodular regenerative hyperplasia after treatment with trastuzumab emtansine. J Clin Oncol. 2016;34(3):e9-12.

12 Prochaska LH, Damjanov I, Ash RM, Olson JC, Khan QJ, Sharma P. Trastuzumab emtansine associated nodular regenerative hyperplasia: a case report and review of literature. Cancer Treat Commun. 2016;5:26-30.

13 Lepelley M, Allouchery M, Long J, Boucherle D, Ranchoup Y, Le Marc'Hadour F, et al. Nodular regenerative hyperplasia induced by trastuzumab emtansine: role of emtansine? Ann Hepatol. 2018;17(6):1067-71.

14 Hassan C, Correal F, Vézina G, Yelle L, Adam JP. Safety of trastuzumab after trastuzumab emtansine-induced nodular regenerative hyperplasia: a case report. J Oncol Pharm Pract. 2020;26(7):1780-4

15 Giordano SH, Temin S, Kirshner JJ, Chandarlapaty S, Crews JR, Davidson NE, et al. Systemic therapy for patients with advanced human epidermal growth factor receptor 2-positive breast cancer: American society of clinical oncology clinical practice guideline. J Clin Oncol. 2014;32(19):2078-99.
16 Morris JM, Oien KA, McMahon M, Forrest EH, Morris J, Stanley AJ, et al. Nodular regenerative hyperplasia of the liver: survival and associated features in a UK case series. Eur J Gastroenterol Hepatol. 2010 Aug;22(8): $1001-5$.

17 Ueno S, Tanabe G, Sueyoshi K, Yoshinaka H Yamamoto S, Kurita K, et al. Hepatic hemodynamics in a patient with nodular regenerative hyperplasia. Am J Gastroenterol. 1996;91: 1012-5.

18 Simsek M, Meijer B, Ramsoekh D, Bouma G, van der Wouden EJ, den Hartog B, et al. Clinical course of nodular regenerative hyperplasia in thiopurine treated inflammatory bowel disease patients. Clin Gastroenterol Hepatol. 2019;17(3):568-70.

19 Breen DP, Marinaki AM, Arenas M, Hayes PC. Pharmacogenetic association with adverse drug reactions to azathioprine immunosuppressive therapy following liver transplantation. Liver Transpl. 2005;11:826-33.

20 Yan H, Endo Y, Shen Y, Rotstein D, Dokmanovic M, Mohan N, et al. Ado-trastuzumab emtansine targets hepatocytes via human epidermal growth factor receptor 2 to induce hepatotoxicity. Mol Cancer Ther. 2016;15: $480-90$. 